\title{
Mudança de Longo Prazo e Regionalização da Evapotranspiração de Referência no Nordeste Brasileiro
}

\author{
Rodrigo Lins da Rocha Júnior ${ }^{1}$ (D), Fabrício Daniel dos Santos Silva ${ }^{1}$ (D), \\ Rafaela Lisboa Costa $^{1}$ (D), Heliofábio Barros Gomes ${ }^{1}$ (D), Helber Barros Gomes ${ }^{1}$ (D), \\ Maria Cristina Lemos da Silva ${ }^{1}$ (D), David Duarte Cavalcante Pinto ${ }^{1}$ (D), Dirceu Luís Herdies ${ }^{2}$ (D), \\ Jório Bezerra Cabral Júnior ${ }^{3}$ (D), Oscar Pita-Díaz ${ }^{4}$ \\ ${ }^{1}$ Instituto de Ciências Atmosféricas, Universidade Federal de Alagoas, Maceió, AL, Brasil. \\ ${ }^{2}$ Centro de Previsão do Tempo e Estudos Climáticos, Instituto Nacional de Pesquisas Espaciais, \\ Cachoeira Paulista, SP, Brasil. \\ ${ }^{3}$ Instituto de Geografia, Desenvolvimento e Meio Ambiente, Universidade Federal de Alagoas, \\ Maceió, AL, Brasil. \\ ${ }^{4}$ Subcoordinación de Hidrología Superficial, Insitituto Mexicano de Tecnología del Agua, \\ Jiutepec, Morelos, Mexico.
}

Recebido em: 13 de Agosto de 2020 - Aceito em: 24 de Setembro de 2020

\begin{abstract}
Resumo
A Evapotranspiração (ET) é a segunda variável mais importante do ciclo hidrológico e influencia inúmeros processos da atmosfera. Desta forma, é interessante estudar as mudanças desta variável sob o contexto das mudanças climáticas antropogênicas e da constante ocorrência de secas no Nordeste Brasileiro (NEB). Foram analisadas as tendências da ET no NEB entre 1980 e 2016 a partir de dados em grade com alta resolução de $0.25^{\circ}$ x $0.25^{\circ}$, constituindo 2071 pontos no NEB. Uma análise de agrupamentos mostrou que é plausível dividir a região em quatro grupos homogêneos. O grupo 1 é referente a área do semiárido na porção central do NEB. O grupo 2 é a faixa que circunda o semiárido. O grupo 3 localiza-se ao norte do Maranhão e o 4 na costa e noroeste do NEB. Analisando-se a tendência média da ET, observa-se que os grupos 2, 3 e 4 apresentaram tendências significativas de aumento da ET de 2,7 mm/ano, 3,51 mm/ano e 2,57 mm/ano, respectivamente. Em análise ponto por ponto, a porção central do NEB e semiárido apresentaram tendências positivas de ET anual ( $\sim 0.8 \mathrm{~mm} / \mathrm{ano})$, enquanto o litoral noroeste e uma parte da região central da Bahia apresentaram tendências negativas $(\sim 1 \mathrm{~mm} / \mathrm{ano})$. Tais resultados devem estar associados as recentes tendências de temperatura e chuvas observadas no NEB, com impactos importantes nos núcleos de desertificação observados em muitas áreas da região, servindo de alerta a gestores ambientais e de recursos hídricos.
\end{abstract}

Palavras-Chave: evapotranspiração, análise de cluster, análise de tendências, teste de Mann-Kendall.

\section{Long-Term Change and Regionalization of Reference Evapotranspiration in the Brazilian Northeast}

\begin{abstract}
Evapotranspiration (ET) is the second most important variable in the hydrological cycle and influences several atmospheric processes. Therefore, the scientific interest in studying changes in that variable under the context of climate change and the usual occurrence of droughts in the Northeast of Brazil (NEB) is particularly valid. ET trends in the NEB were analysed from high resolution grid data $\left(0.25^{\circ} \times 0.25^{\circ}\right)$ for the period between 1980 and 2016 , comprising 2071 points in the NEB. A cluster analysis has shown that the region is climatically divisible into four homogenous groups. Group 1 represents the semiarid area in the central portion of the NEB. Group 2 is formed by the non-contiguous lands that surround the semiarid region. Group 3 is located in the north of Maranhão, whereas Group 4 is com-
\end{abstract}

Autor de correspondência: Rodrigo Lins da Rocha Júnior, rrodrigo.junior@hotmail.com. 
posed of patches spreading mostly over the eastern coast and the northwest of the NEB. By analysing the mean ET trend, it is possible to observe that groups 2, 3 and 4 show significant upward trends of $2.7 \mathrm{~mm} /$ year, $3.51 \mathrm{~mm} /$ year and $2.57 \mathrm{~mm} /$ year, respectively. According to a step-by-step analysis, the central (semiarid) area of the NEB presented a positive ET trend $(\sim 0.8 \mathrm{~mm} /$ year), whilst the northwestern coast and part of central Bahia showed negative trends $(\sim-1$ $\mathrm{mm} /$ year). Such results may be linked to recent temperature and rainfall trends observed in the NEB, with important impacts for the desertification nuclei observed in many parts of the region, which represent a warning for environmental and water resources managers.

Keywords: evapotranspiration, cluster analysis, trend analysis, Mann-Kendall test.

\section{Introdução}

A evapotranspiração (ET) da superfície é um processo crucial para o sistema climático, tendo influência sobre o ciclo hidrológico, ciclo do carbono e troca de energia entre a superfície e atmosfera (Oki e Kanae, 2006; Jung et al., 2010). É a segunda variável mais importante no ciclo hidrológico depois da precipitação. A ET devolve a atmosfera $60 \%$ de toda água precipitada sobre o solo e sua variação pode influenciar a precipitação e a disponibilidade hídrica em rios e lagos (Zeng et al., 2014; Zhang et al., 2016; Jin et al., 2017). Variações da ET influencia também o fluxo de calor latente, que impacta no calor sensível e temperatura da superfície e por consequência provoca mudanças significativas no clima regional e global (Vourtilis et al., 2002; Jung et al., 2010; Tabari et al., 2011; Jin et al., 2017; Liu et al., 2019; Santos et al., 2020). Portanto, entender os padrões, magnitudes e mudanças a longo prazo de ET pode ser crucial para lidar com problemas de hidrologia, ecologia, e gerenciamento de recursos hídricos em regiões áridas e semiáridas.

Mudanças em algumas variáveis meteorológicas no contexto das mudanças climáticas, como temperatura, umidade relativa e velocidade do vento, impactam diretamente na ET (Silva et al., 2018). Eventualmente, tais mudanças climáticas podem provocar o aumento de condições de seca por todo o globo, devido ao aumento da ET, agravando o processo de desertificação em regiões áridas e semiáridas (Jung et al., 2010). Estudos recentes apontam para um aumento da temperatura global e de extremos de precipitação (Fischer e Knutti, 2015; Alexander, 2016), tais resultados estão em parte associados a variações de ET da superfície, como em Chattopadhyay e Hulme (1997), que mostraram aumento da temperatura na Índia em contraste com a diminuição da ET. Além disso, o aumento da umidade relativa e diminuição da radiação estão correlacionados com ET: Thomas (2000) mostrou diminuição da ET em todas as estações do ano no nordeste e sudeste da China; Tabari et al. (2010) encontrou tendências positivas em $70 \%$ das estações analisadas no Irã; Douvile et al. (2012) reportou o aumento conjunto da precipitação e ET em todo o globo; Jin et al. (2017) detectou tendências positivas de ET sobre o Loess Plateau, na região central da China.

Alguns trabalhos estudaram de formas diversas a ET sobre o domínio do Brasil (Vourlitis et al., 2002; Sanches et al., 2011). Moraes et al. (1998) encontrou tendências positivas de precipitação e ET na Bacia do rio Piracicaba na região sudeste do Brasil. Tarso et al. (2014) avaliou a tendência das componentes do balanço hídrico no domínio do bioma Cerrado, encontrando tendências significantes da ET. Silva et al. (2018) avaliou a tendência da evapotranspiração potencial no Estado de Alagoas e encontrou aumento significativo da variável na porção ocidental do Estado. Apesar desses trabalhos, até o momento, nenhuma análise de tendência da ET foi realizada focando todo o domínio do NEB (Paulino et al., 2019). No contexto das mudanças climáticas, estudos estão sendo gradualmente movidos a avaliar outras variáveis atmosféricas relevantes ao ciclo hidrológico para entender os impactos das mudanças climáticas (Douville et al., 2012).

O objetivo primário deste trabalho é avaliar as tendências de longo prazo da ET sobre o NEB, a nível anual e sazonal, utilizando dados de alta qualidade e grande resolução espacial (Xavier et al., 2016). O objetivo secundário é estabelecer de forma objetiva as regiões homogêneas de ET sobre o NEB utilizando métodos robustos de agrupamento. Os resultados deste trabalho podem ser de grande valia para compreender alterações no ciclo hidrológico de toda região e fornecer resultados consistentes para ações estratégicas por parte dos gestores de recursos hídricos. $\mathrm{Na}$ sessão 2 detalha-se a região de estudo, fonte de dados e metodologia. Na sessão 3 apresenta-se os resultados e discussões referentes a outros trabalhos relacionados. Por fim, na sessão 4, expõe-se as conclusões.

\section{Materiais e Métodos}

\subsection{Dados e área de estudo}

O NEB está localizado na zona equatorial, entre 1$21^{\circ} \mathrm{S}$ e $32-49^{\circ} \mathrm{W}$, com $1.800,555 \mathrm{~km}^{2}$, correspondendo a aproximadamente $20 \%$ do território brasileiro. O NEB é composto por nove estados e 1794 municípios (Fig. 1a). A Mata Atlântica é a vegetação presente na costa leste e a floresta amazônica no extremo oeste do NEB. Na porção central (região semiárida), a vegetação presente é a Caatinga. A porção de transição entre a Caatinga e a Mata Atlântica é denominada Agreste. Variados sistemas meteorológicos associados a topografia do NEB, causam grande variabilidade climática interanual (Rodrigues et al., 2011; Carvalho et al., 2013; da Rocha Júnior et al., 2019; da Rocha Júnior et al., 2020). 


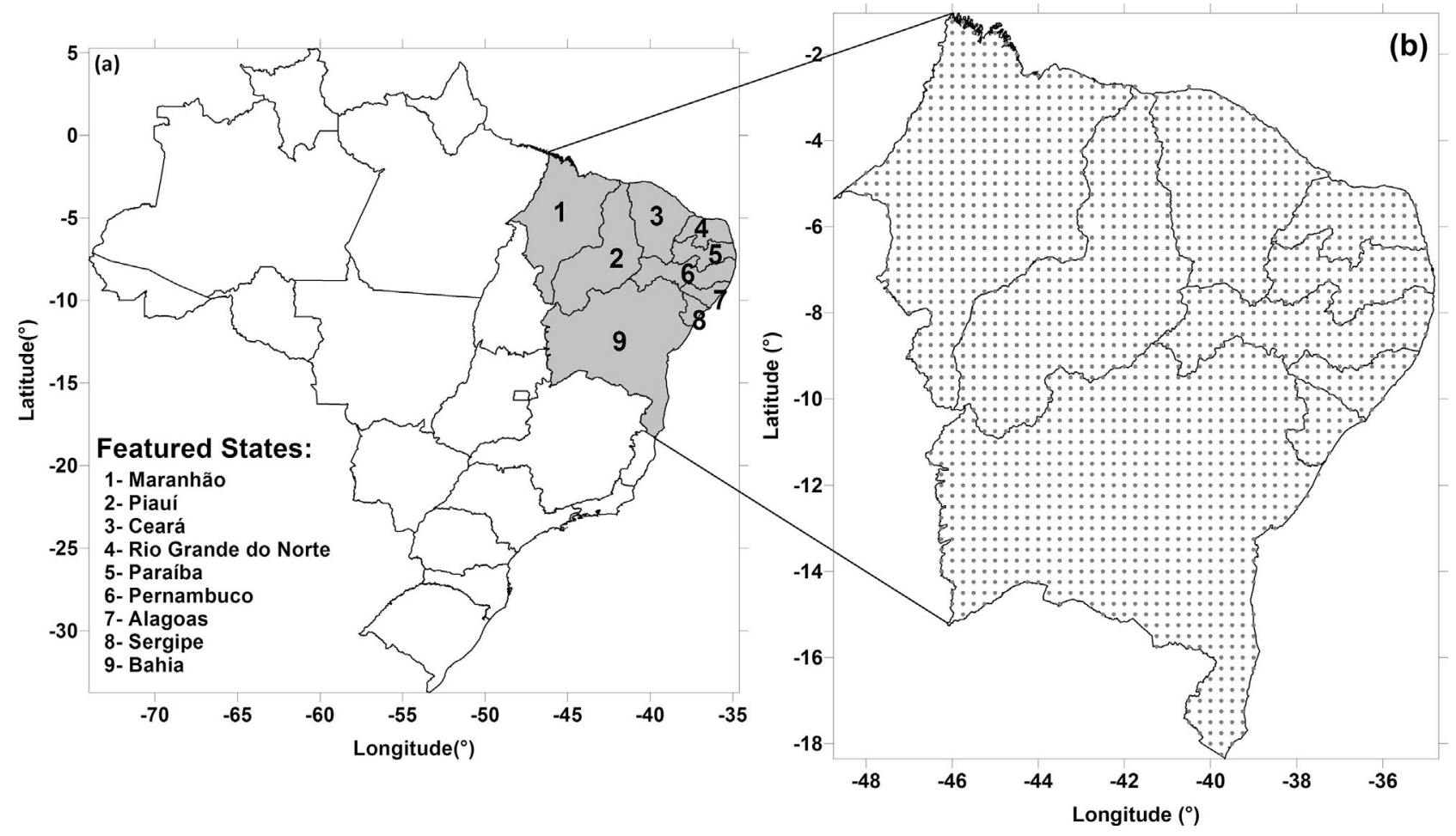

Figura 1 - (a) Mapa do Brasil destacando a região Nordeste em cinza, com estados numerados e identificados e (b) espaçamento de grade com resolução de $0,25^{\circ} \times 0,25^{\circ}$. Cada círculo em cor cinza se refere a um ponto de grade na área, totalizando 2071 pontos espacialmente distribuídos no NEB.

Foram utilizados dados de ET fornecidos por Xavier et al. (2016). Essa análise em grade possui uma resolução espacial de $0,25^{\circ} \times 0,25^{\circ}$ de várias variáveis meteorológicas para todo o Brasil no período de 1980 a 2016 (Fig. 1b). A ET foi calculada pelo método de PenmanMonteith (PM) (Allen et al., 1998; Raes, 2012), com base em dados de temperaturas máxima e mínima, radiação solar, umidade relativa e velocidade do vento a $2 \mathrm{~m}$. O método PM foi amplamente verificado e validado com base em dados de lisímetros de várias regiões climáticas (Allen et al., 1994, Ventura et al., 2000, López-Urrea et al., 2006). Para a construção de séries temporais de ET, Xavier et al. (2016) utilizou o método de interpolação Angular Distance Weighting (ADW).

Os dados disponíveis por Xavier et al. (2016) vem sendo amplamente utilizados em estudos climáticos recentes, cuja qualidade foi comprovada mediante comparações com dados de estações de superfície e derivadas de sensoriamento remoto: Paredes-Trejo et al. (2019) utilizaram os dados de precipitação disponibilizados por Xavier et al. (2016) para avaliar o desempenho das estimativas de precipitação derivadas de produtos de umidade do solo via satélite com base em micro-ondas; Silva et al. (2018; 2019) analisaram índices de extremos climáticos de precipitação na Amazônia e no NEB; da Rocha Júnior et al. (2019) analisaram tendências em quantis extremos do índice de seca SPEI (Standard Precipitation and Evapotranspiration Index) no NEB; Andrea (2019) usaram dados de Xavier et al. (2016) como base de referência para avaliar cenários futuros de mudanças climáticas e impactos na produção de milho no estado do Mato Grosso; dos Reis et al. (2020a; 2020b) verificaram a alternância de períodos secos e úmidos favoráveis a produção de soja na área do MATOPIBA, além de estudarem as tendências relativas a extremos de precipitação e temperaturas.

\subsection{Cluster analysis}

Cluster analysys, também conhecida como análise de agrupamento, permite classificar uma amostra de dados em grupos homogêneos e com máxima diferença e heterogeneidade entre cada grupo (Carvalho et al., 2016). Foram utilizados dois métodos distintos para determinar regiões homogêneas de ET no NEB, um método hierárquico (Ward) e um método não hierárquico (K-means). Diversos estudos na área de hidrologia e climatologia aplicaram ambos os métodos em variáveis meteorológicas (Liu et al., 2015; Regonda et al., 2016; Carvalho et al., 2016; Raziei 2017; Ahmadi et al., 2017; Costa et al., 2020). Métodos hierárquicos tem como uma de suas características o cálculo para o número de grupos considerado ideal (Seidel et al., 2018). Até então, muitos trabalhos escolhem o número de grupos de forma subjetiva (Zhang et al., 2016). A vantagem de se trabalhar com os dois métodos de análise de cluster, Ward e K-means, é minimizar diferenças espaciais que podem ser consideráveis devido ao número de grupos 
estabelecidos, obtidos inicialmente pelo método de Ward e aplicado também ao método K-means, que realmente podem diferir muito em área quando se atribui subjetivamente poucos ou muitos grupos aos dados analisados. Visando obter um número de grupos condizentes com padrões reais de ET sobre o NEB, aplicamos o método de Silhouettes para determinar de forma objetiva o número de grupos selecionado. Detalhes mais específicos sobre o método pode ser encontrado em Rousseeuw (1987), e sobre o algoritmo dos métodos de Ward e K-means podem ser encontrados em Wilks (2011).

Segundo Maciel et al. (2015), Além de permitir visualizar graficamente os agrupamentos, o gráfico de determinado cluster $C$ é composto por um valor $s(i), i=1, \ldots$, $n$, que reflete a qualidade da alocação dos elementos dos grupos. Cada elemento do cluster pode ser representado por $i$. Para cada objeto $i$, o valor $s(i)$ é calculado pela Eq. (1):

$$
s(i)=\frac{b(i)-a(i)}{\max (a(i), b(i))}
$$

onde $a(i)$ é a dissimilaridade média do objeto i em relação a todos os objetos do mesmo grupo $C$, e $b(i)$ é a dissimilaridade média entre o objeto $i$ em relação a todos os objetos do grupo vizinho mais próximo a ele, grupo $X$.

O valor de $s(i)$ varia no intervalo entre -1 e 1 , sendo adimensional. Quando um valor de $s(i)$ ö 1 , significa que o objeto $i$ foi bem classificado no grupo $C$, pois $a(i)<b(i)$. Se o valor de $s(i) \ddot{~ o ̈ ~}-1$, significa que o objeto foi mal classificado, pois $a(i)>b(i)$, ou seja, o objeto $i$, em média, está mais distante dos objetos do seu próprio grupo, isto é, o objeto do grupo $C$ está mais próximo dos objetos do grupo $X$. Por sua vez, se $s(i)$ ö 0 , o objeto $i$ está entre os grupos $C$ e $X$, isso ocorre quando $a(i)=b(i)$, indicando que o objeto está num ponto intermediário a dois grupos. Logo, quanto mais próximo a 1 , melhor será a qualidade do agrupamento (Souza, 2007; Maciel et al., 2015).

\subsection{Teste de tendência de Mann-Kendall}

$\mathrm{O}$ teste não paramétrico de Mann-Kendall (MK) foi aplicado para testar a significância estatística das tendências nas séries anuais e sazonais de ET. O teste MK (Mann, 1945; Kendall, 1975) mostra se as tendências estatísticas são aleatórias ou não, sem a necessidade de conhecimento prévio da distribuição dos dados (Sem, 1968; Richard, 1987; Salmi et al., 2002; Santos et al., 2016; Silva et al., 2018; Costa et al., 2020), sendo considerado o mais adequado para analisar variações em séries temporais climatológicas (Onoz e Bayazit, 2003; Haylock et al., 2006; Dufek e Ambrizzi, 2008; Machiwal e Jha, 2008; Someé et al., 2013). De acordo com o teste, a hipótese nula $\mathrm{H} 0$ assume que não há tendência. Ou seja, a série é independente e aleatoriamente ordenada. A hipótese alternativa $\mathrm{H} 1$ assume que há uma tendência monotônica (Par- tal e Kahya, 2006; Satyamurty et al., 2008). O teste MK geralmente é aplicado considerando a estatística $\mathrm{S}$, como em Modarres e da Silva (2007) na Eq.(2).

$$
S=\sum_{i=2}^{n} \sum_{j=1}^{i-1} \operatorname{sign}\left(x_{i}-x_{j}\right)
$$

onde $X_{i}$ é o valor sequencial dos dados, $n$ é o tamanho da série temporal e $\operatorname{sign}\left(X_{i}-X_{j}\right)=-1$ se $\left(X_{i}-X_{j}\right)<0 ; \operatorname{sign}\left(X_{i}-\right.$ $\left.X_{j}\right)=0 \operatorname{se}\left(X_{i}-X_{j}\right)=0$ e $\operatorname{sign}\left(X_{i}-X_{j}\right)=1$ se $\left(X_{i}-X_{j}\right)>0$. A hipótese nula $\mathrm{H}_{0}$ é que uma amostra de dados $\left\{X_{t}: t=1,2\right.$, ..., $n\}_{i}$ é independente e identicamente distribuído. A hipótese alternativa $\mathrm{H}_{1}$ é que existe uma tendência monotônica em $\left\{X_{t}\right\}$. Cada par de valores observados $\left(X_{i}, X_{j}\right)$ onde $i>j$ é inspecionada para encontrar $X_{i}>X_{j}$ (primeiro tipo) ou $X_{i}$ $<X_{j}$ (segundo tipo). A esperança $E[S] e$ variância $\operatorname{Var}[S]$ da estatística $S$ são obtidas pelas Eqs. (3) e (4):

$$
E[S]=0
$$

$$
\operatorname{Var}[S]=\frac{n(n-1)(2 n+5)-\sum_{p=1}^{q} t_{p}\left(t_{p}-1\right)\left(2 t_{p}+5\right)}{18}
$$

em que $n$ é o número de observações e, considerando o caso em que a série pode ter grupos com observações iguais, $P$ é o número de grupos com observações iguais e $t_{P}$ é o número de observações iguais no grupo ${ }_{P}$ Um distribuição normal da variável $Z$ é construída como mostram as Eqs. (5):

$$
Z=\left\{\begin{array}{l}
\frac{S-1}{\sqrt{\operatorname{Var}(S)}}, S>0 \\
0, S=0 \\
\frac{S+1}{\sqrt{\operatorname{Var}(S)}}, S<0
\end{array}\right.
$$

Em um teste duplo para a tendência, a hipótese nula de não tendência é rejeitada se $|Z|>Z_{a / 2}$, onde $\alpha$ é o nível de significância. Dada a grande densidade dos dados, e com objetivo de encontrar maiores áreas com tendências uniformes, consideramos, como em algumas pesquisas, tendências estatisticamente significantes quando o $\mathrm{p}$-valor for igual ou menor a 10\% (Grieser et al., 2002; Staeger et al., 2003; del Río et al., 2007; Ghafouri-Azar et al., 2018; Dinpashoh e Babamiri, 2020). Para mais detalhes sobre o método MK, recomenda-se a leitura de Wilks (2011).

O software R versão 3.6.1 foi utilizado em todas as etapas deste trabalho: tratamento dos dados, análise de cluster, cálculo das tendências, assim como para a geração dos gráficos e mapas apresentados nos resultados e discussões. 


\section{Resultados e Discussões}

\subsection{Grupos de ET}

O método de Silhouettes foi aplicado para determinar objetivamente o número ideal de clusters para o NEB. $\mathrm{O}$ número ideal apontado pelo método é $\mathrm{k}=4$ (Fig. 2a). $\mathrm{O}$ valor de $\mathrm{k}$ foi considerado para os dois métodos de clusterização aplicados, K-means e Ward (Figs. 2b e 2c). Ao aplicar as técnicas, obteve-se padrões semelhantes para ambos os métodos. A área do cluster 1 apresenta uma delimitação que abrange a maior parte da área do semiárido do NEB (Fig. 2b e 2c). O cluster 2 por sua vez é referente a uma área que cerca o semiárido pelo leste e oeste. Uma pequena área classificada no cluster 2 está presente no interior norte da região do semiárido, e também avança consideravelmente em direção ao oeste do estado do Maranhão. O método de Ward também classificou para o cluster 2 uma pequena área no interior sul do semiárido (Fig. 2c). A área do cluster 2 no lado leste é característica da sub-região do NEB denominada de Zona da Mata. O cluster 3 abrange pequenas áreas do litoral leste e uma área na porção noroeste do NEB, ao norte do Maranhão. Na porção noroeste do NEB se encontra o cluster 4, em que sua área varia em função do método de agrupamento usado. No método de k-means a área do cluster 4 é mais ampla que a caracterizada pelo método de Ward, desde parte do norte piauiense até o centro-norte maranhense.

\section{a) Optimal number of clusters \\ a) Silhouette method}
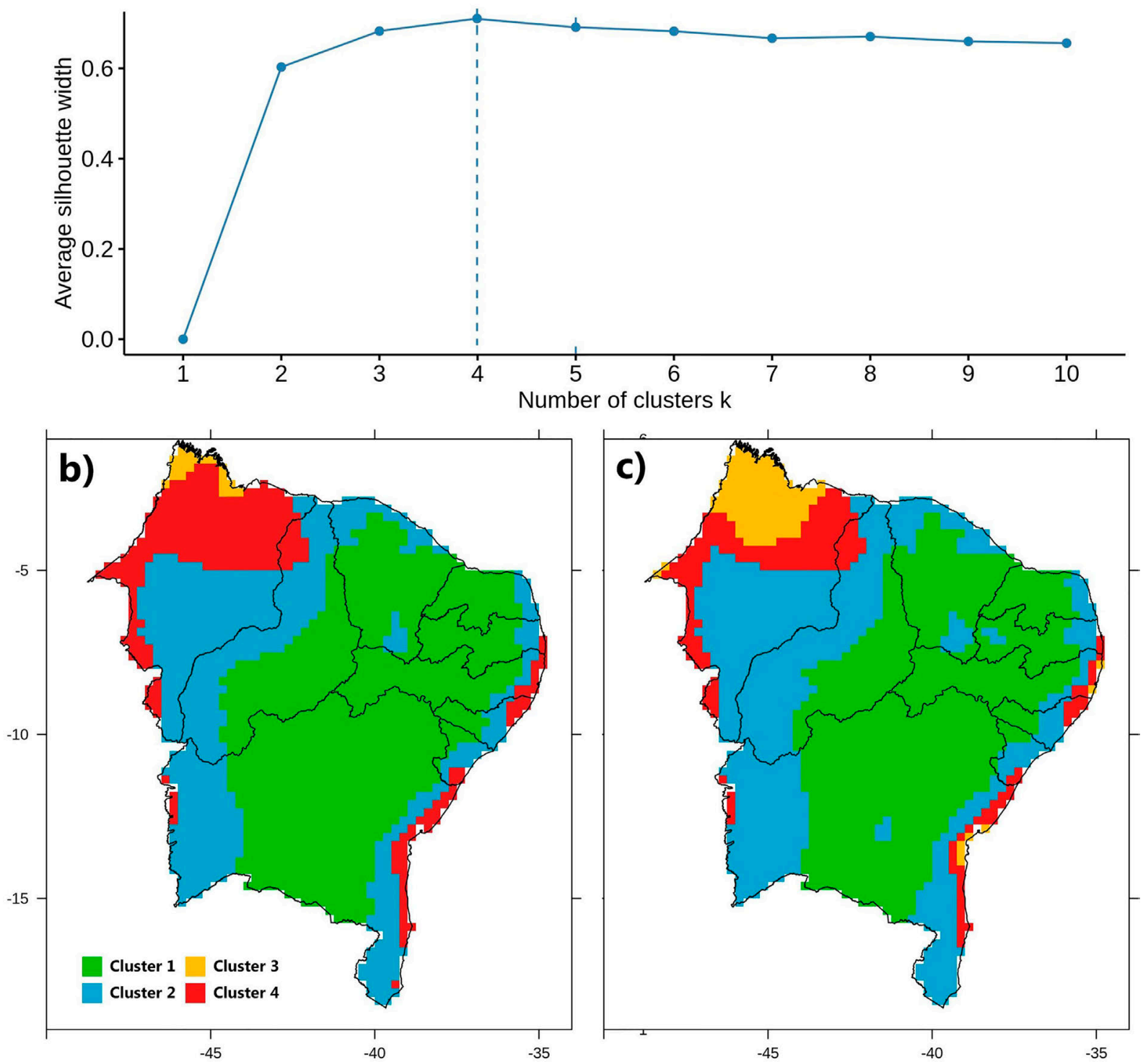

Figura 2 - Determinação dos grupos de ET no NEB pela aplicação do método de Silhouette (a), k-means (b) e Ward (c). 


\subsection{Tendências dos grupos}

Uma vez definidas de forma objetiva as regiões homogêneas de ET no NEB, calculou-se a tendência média da variável para cada grupo. No geral, observam-se tendências positivas em todos os grupos (Fig. 3). O grupo 1 referente ao semiárido mostra uma tendência positiva de $1,48 \mathrm{~mm} / \mathrm{ano}$, que não obteve significância estatística (pvalor $=0.24)($ Fig. 3a). Os grupos 2, 3 e 4 obtiveram ten-
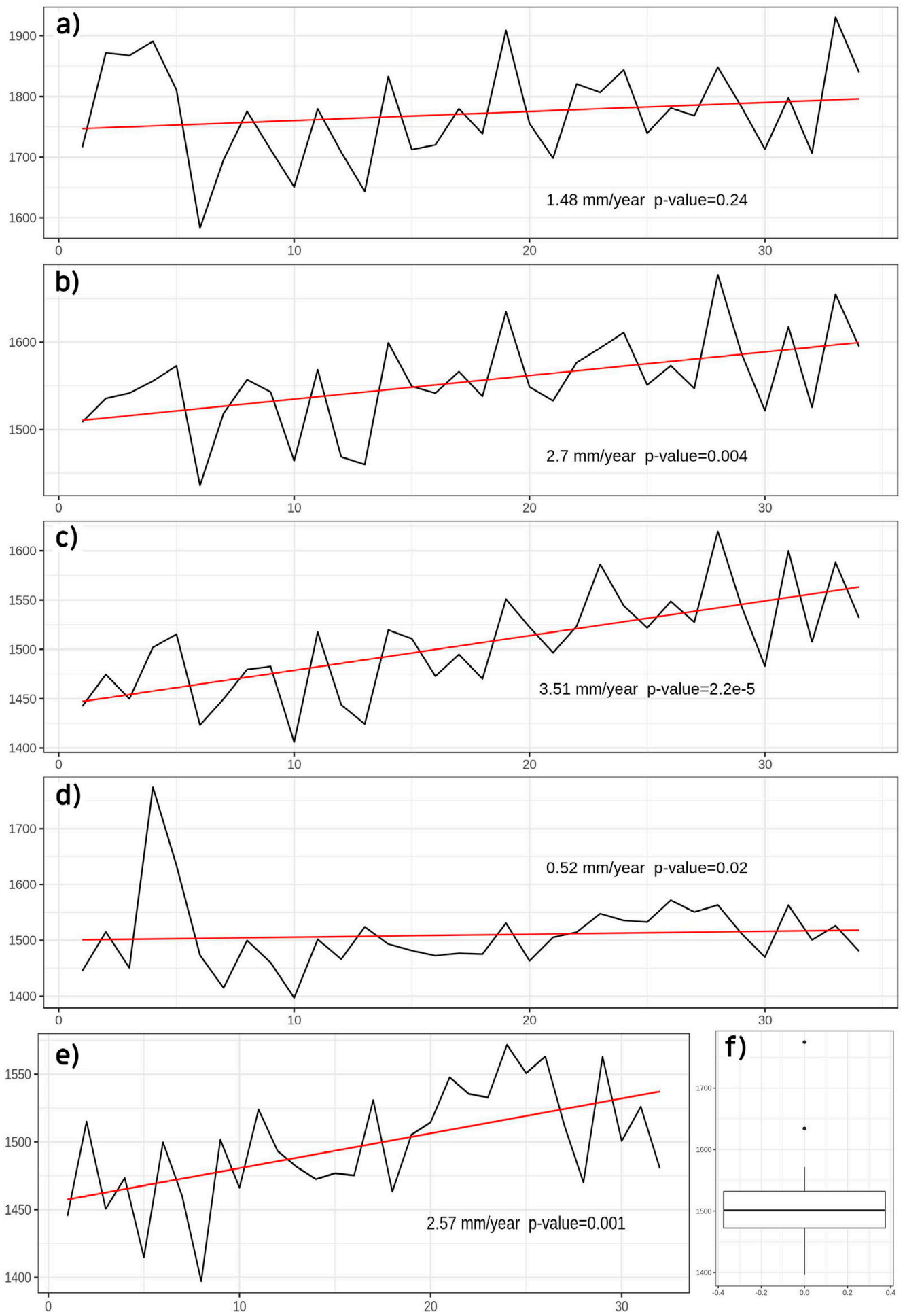

Figura 3 - Tendência geral dos grupos definidos na análise de agrupamento. (a) Tendência do grupo 1, (b) tendência do grupo 2, (c) tendência do grupo 3 , (d) tendência do grupo 4, (e) tendência do grupo 4 sem outliers, (f) boxplot da série referente ao grupo 4 exibindo a existência de dois outliers. 
dências significativas de $2,7 \mathrm{~mm} / \mathrm{ano}, 3,51 \mathrm{~mm} /$ ano e $0,52 \mathrm{~mm} / \mathrm{ano}$, respectivamente (Fig. $3 \mathrm{~b}$ e $3 \mathrm{~d}$ ). Dados espúrios referentes a um conjunto localizado de 10 pontos de grade foram identificados na série do grupo 4 e foram retirados das estimativas de tendências (Fig. 3f), por provocarem erros indesejados ao cálculo. Uma vez excluídos os valores espúrios (caracterizados por apresentarem valores médios diários de velocidade do vento muito superiores a seus vizinhos), as tendências foram recalculadas, com o grupo 4 mostrando tendência positiva mais acentuada da ordem de 2,57 mm/ano (Fig. 3e). Apesar das tendências médias de todos os grupos serem positivas, é necessário analisar os padrões espaciais das tendências de ET para caracterizar mudanças locais da variável.

\subsection{Padrões espaciais de tendências de ET}

A tendência anual da ET diária é mostrada na Fig. 4. Uma grande área de tendência positiva, variando de $0,5 \mathrm{a}$ $1,2 \mathrm{~mm} / \mathrm{ano}$, é observada na porção sudoeste do NEB abrangendo parte dos Estados da Bahia, Piauí e Maranhão. Na região central também observa-se tendências positivas $(\sim 0,5 \mathrm{~mm} / \mathrm{ano})$ sobre parte dos Estado da Bahia, Piauí e Pernambuco. Tendências positivas de ET estão de acordo com a perspectiva de intensificação do ciclo hidrológico em resposta ao aquecimento global (Huntington, 2006; Douville et al., 2012). O litoral do NEB do Ceará até a Bahia apresenta tendências negativas de ET que variam de $-0,1$ a $-1,4 \mathrm{~mm} / \mathrm{ano}$. Uma área de tendência negativa é observada no interior da Bahia. O litoral é a zona de maior desenvolvimento econômico e social de toda o NEB, portanto, uma diminuição da ET nessa zona pode estar associado ao maior armazenamento de água no solo ou à

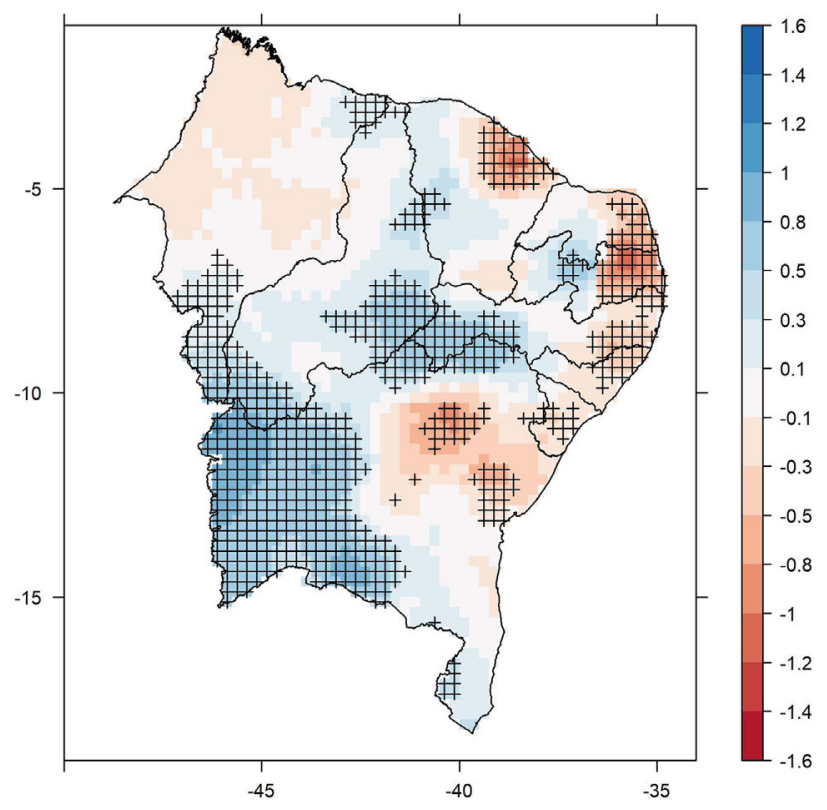

Figura 4 - Mapa de tendência da ET anual em todo o território do NEB. A área hachurada indica significância estatística ao nível de $90 \%$. descontinuidades no ciclo hidrológico devido ao crescimento de zonas urbanas na região.

A Fig. 5 apresenta as tendências de ET diária para as estações do ano. $\mathrm{O}$ trimestre dezembro-janeiro-fevereiro (DJF), representativo do verão, apresenta tendências semelhantes ao observado na figura anterior (Fig. 4). Há tendências positivas no sudeste e interior do NEB variando entre 0,5 a $1,2 \mathrm{~mm} / \mathrm{ano}$. DJF abrange a maior parte do período chuvoso do centro-oeste do NEB. No mesmo período se observa tendências negativas em parte do litoral norte, sobre porções dos Estados do Ceará, Rio Grande do Norte e Paraíba. Os valores de tendência variam de $-0,3$ a $-1,6 \mathrm{~mm} / \mathrm{ano}$. O período DJF abrange também o início da estação chuvosa do norte do NEB, quando a Zona de Convergência Intertropical (ZCIT) se dispõe mais ao sul da sua posição média (Cavalcante, 2010). A outra metade do litoral norte apresenta uma pequena região com tendência positiva entre o Estado do Maranhão e Piauí.

O trimestre março-abril-maio (MAM), representativo do outono, exibe decaimento das tendências significantes observadas no período DJF (Fig. 5b). O sudoeste do NEB apresenta uma área menor de tendências positivas, porém ainda considerável. $\mathrm{O}$ interior e norte do NEB apresentam poucos pontos com tendência significativa. $\mathrm{O}$ trimestre MAM compreende o mais importante período chuvoso no norte do NEB.

O trimestre junho-julho-agosto (JJA), representativo do inverno, mostra tendências negativas em toda zona leste do NEB até o norte do Estado do Ceará (Fig. 5c). No interior da Bahia se observa tendências negativas bem pronunciadas ( $>-1,4 \mathrm{~mm} / \mathrm{ano}$ ), enquanto no setor oeste do Estado se observa uma área de tendências positivas semelhante ao observado no trimestre MAM. JJA é o período chuvoso da porção leste do NEB e isso se reflete nas tendências observadas.

O trimestre setembro-outubro-novembro (SON), representativo da primavera, mostra intensificação das tendências positivas na porção sudeste do NEB, semelhante ao observado em DJF, porém mais intenso, apresentando tendências entre 0,8 e $1,6 \mathrm{~mm} /$ ano (Fig. $5 \mathrm{~d}$ ). Na porção central do NEB se observa o crescimento máximo da área com tendências positivas $(0,3$ a $1,2 \mathrm{~mm} / \mathrm{ano})$. Por outro lado as tendências do leste do NEB começam a decair, a partir do litoral sul.

No geral o interior do NEB apresenta tendências positivas de ET, que pode estar associada ao aumento da temperatura local e intensificação do processo de desertificação. Costa et al. (2020) mostraram tendências majoritariamente positivas em extremos relacionados à temperatura em boa parte do Nordeste, com acentuado número de estações apresentando significância estatística na porção central do semiárido e oeste da Bahia, além de tendências negativas da precipitação total anual, concordando com as tendências positivas da ET observadas nesta pesquisa. Além disso, corroborando os resultados, Tomasella et al. 
a)

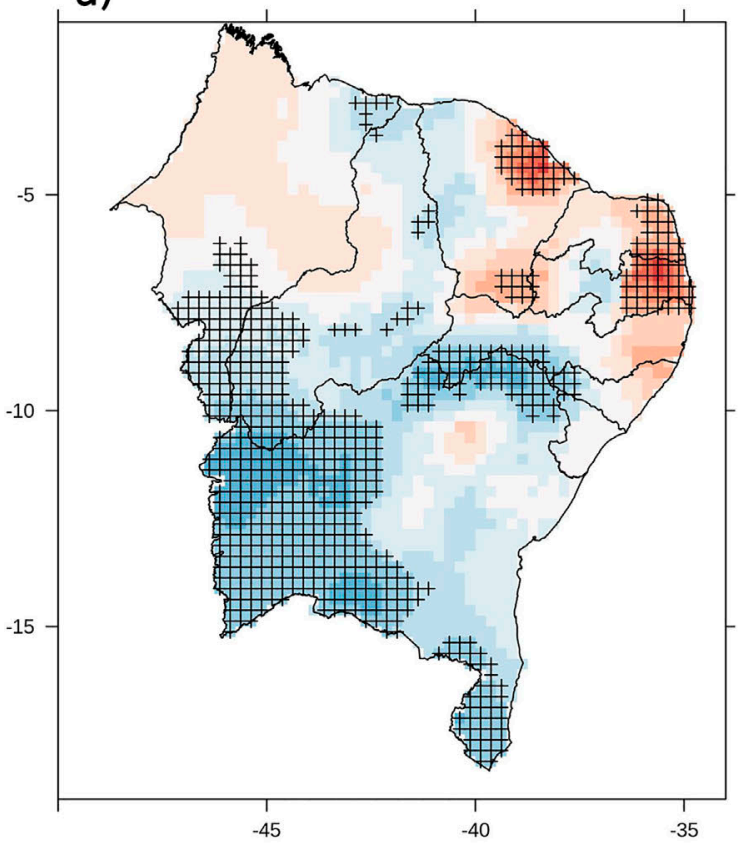

C)

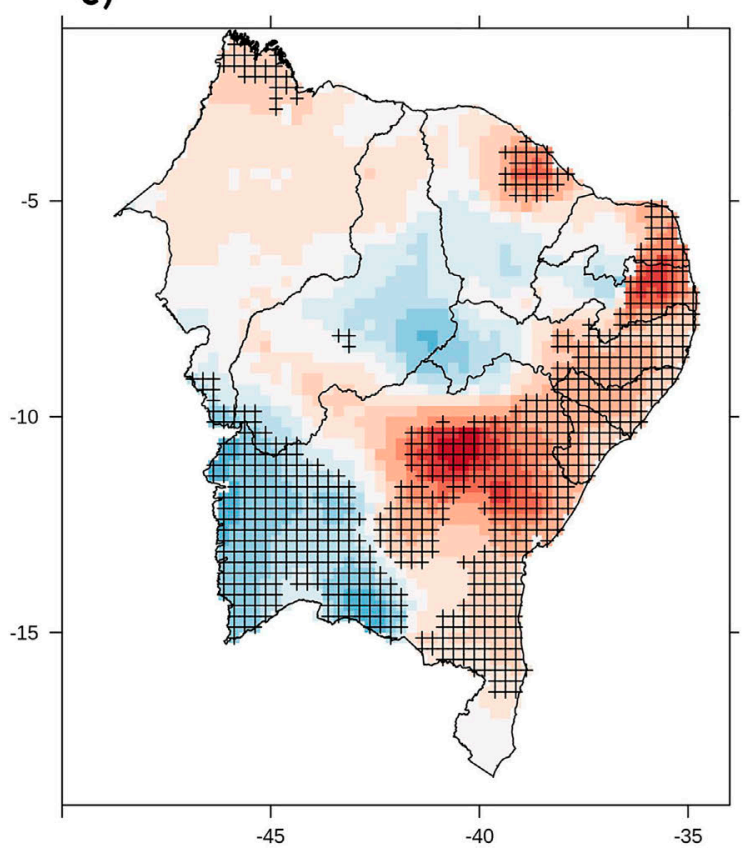

b)

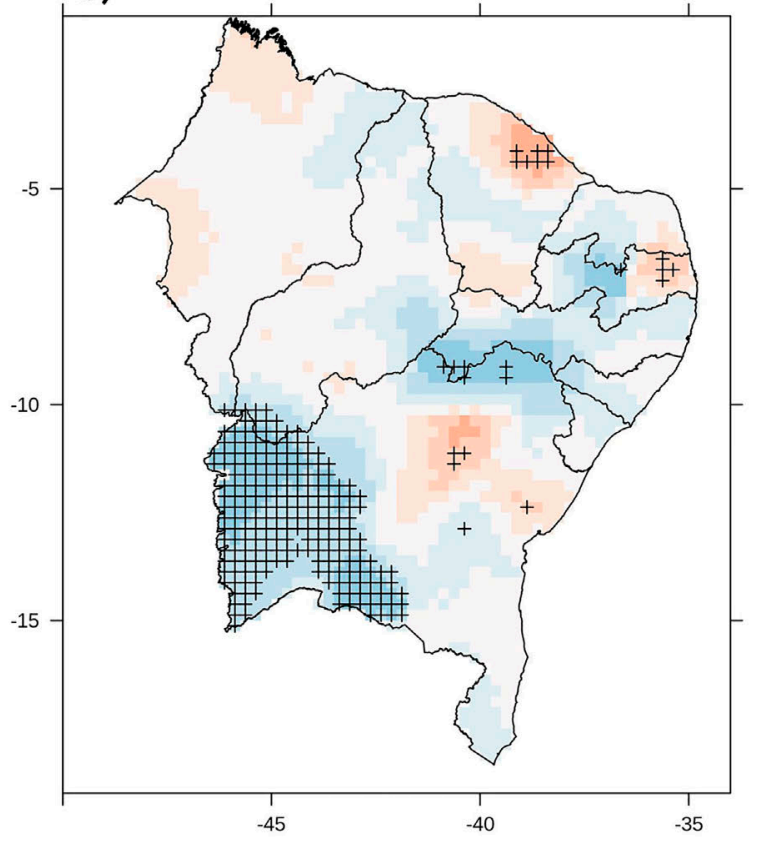

d)

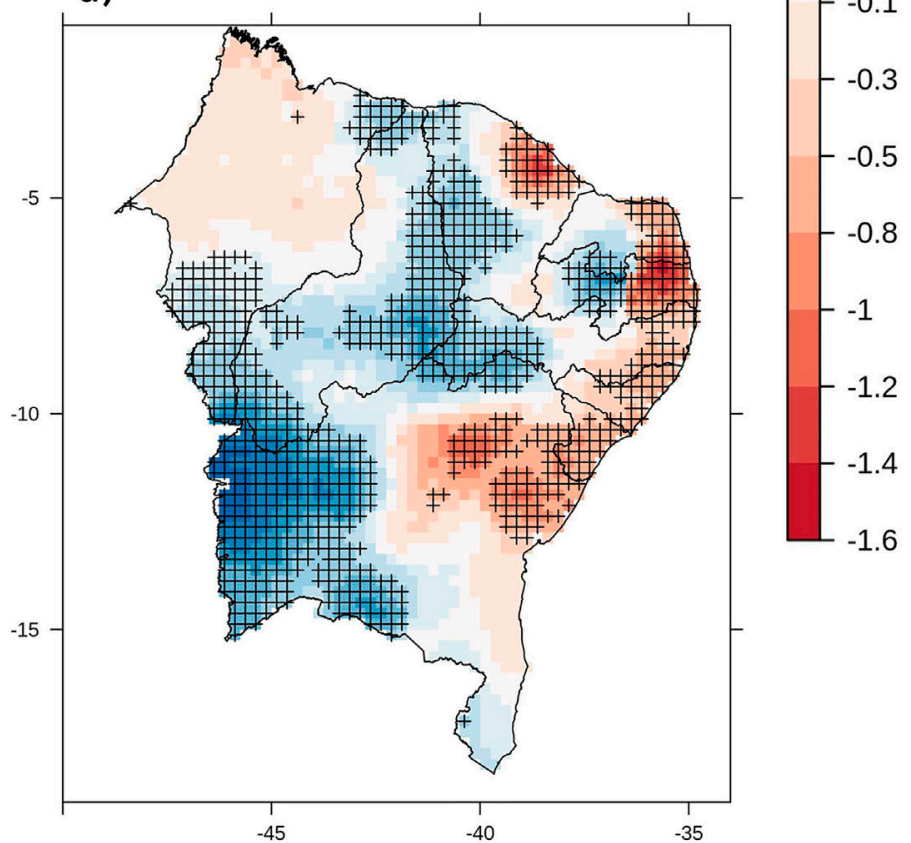

Figura 5 - Mapas de tendências da ET sazonal para o a) Verão, b) Outono, c) Inverno e d) Primavera. A área hachurada indica significância estatística ao nível de $90 \%$.

(2018) evidenciaram grandes áreas com aumento no processo de desertificação, com destaque para a área central do semiárido.

Cabral Júnior et al. (2019) mostraram que tendências negativas da ET são associadas ao aumento da umidade. Os resultados desta pesquisa mostram um leste do NEB com essa característica, o que pode ser confirmado em parte pelo estudo de da Rocha Júnior et al. (2019), onde foi caracterizada tendência positiva nos quantis extremos e central do SPEI-3 no leste do NEB, principalmente nos trimestres novembro-dezembro-janeiro e maio-junho-julho, e negativa apenas no trimestre fevereiro-março-abril. Ainda, Costa et al. (2020) mostraram que muitas estações de superfície no leste do NEB apresentam tendências de aumento nas chuvas extremas de um dia e acumulada em cinco dias, além de aumento no número de dias úmidos 
consecutivos. Vale ressaltar que a intensa seca que atingiu o NEB de 2012 a 2018, cujas características foram discutidas por vários autores (Silva et al., 2013; Cunha et al., 2015; Alvalá et al., 2017; Cunha et al., 2018; Martins et al., 2018a; Martins et al., 2018b; Cunha et al., 2019) pode ter influenciado consideravelmente os resultados encontrados nesta pesquisa.

O aumento da precipitação também é um sinal esperado como resposta ao aquecimento global (Douville et al., 2012). Marengo et al. (2016) analisou cenários gerados por modelos do IPCC no domínio do NEB para avaliar o futuro das secas na região. Os modelos mostraram um aumento na precipitação no verão austral e uma diminuição no inverno austral. Em geral, os modelos apontam para uma maior intensificação da seca no interior do NEB. Jenkins e Warren (2015) analisaram outros cenários usando o índice de seca SPI nas escalas de 6 e 12 meses, em geral, com as projeções também mostrando aumento na intensidade, frequência, duração e magnitude das secas para o NEB. Eterna et al. (2018), a partir de dados observados, encontraram tendências de precipitação positivas para os meses de verão no Estado da Paraíba, e Salviano et al. (2016) apontaram para um incremento da temperatura $\mathrm{e}$ da ET no sul e leste do NEB em quase todos os meses do ano, entre 1961-2011.

\section{Conclusões}

Neste trabalho foram analisados os padrões espaciais da ET no NEB utilizando técnicas objetivas de agrupamento e as respectivas tendências a longo prazo em cada grupo, utilizando uma fonte de dados em grade de alta resolução e qualidade. O teste de Silhouettes aplicado aos dados de ET anual mostrou que o NEB deve ser dividido em 4 grupos homogêneos de ET.

Em nível anual, os resultados mostram tendências positivas significativas em uma grande área no oeste da Bahia e sul do Maranhão e Piauí, além de outra grande área entre o norte da Bahia, oeste de Pernambuco e leste do Piaú. Núcleos menores são observados entre Paraíba, Ceará e Maranhão. Áreas com tendências positivas de ET indicam maior exposição do ambiente a períodos secos mais duradouros e diminuição das chuvas no respectivo período chuvoso, enquanto áreas com tendências negativas como as observadas em áreas do litoral nordestino são prenúncio de tendências positivas em relação ao período chuvoso, como indicado em outros estudos na literatura.

A análise sazonal mostra que as características observadas anualmente têm suas áreas ampliadas ou reduzidas de acordo com a estação do ano, mas no geral mantendo a principal característica observada de aumento da ET no interior do NEB e diminuição no leste do NEB até o Ceará. O estado do Maranhão tem a maior parte de seu território sem tendências significativas. $\mathrm{O}$ pico das tendên- cias na faixa litorânea do NEB é observado no inverno, enquanto os mínimos são observados no outono.

Como discutido, o NEB possui importantes núcleos de desertificação, que em geral se encontram na porção semiárida, onde as tendências positivas da ET estão provavelmente associadas ao aumento das temperaturas e diminuição das chuvas. Contudo, a influência recente de um dos maiores períodos secos já registrados no NEB, de 2012 a 2018, pode ter influenciado na magnitude das áreas de tendências encontradas nessa pesquisa. Faz-se necessário complementar este estudo futuramente, com uma análise de tendências das duas principais componentes da equação de ET pelo método de Penman-Monteith, a radiativa e a aerodinâmica, a fim de identificar o peso de cada uma delas na distribuição temporal da flutuação da ET no NEB.

\section{Agradecimentos}

Os autores agradecem a Coordenação de Aperfeiçoamento de Pessoal de Nível Superior (CAPES) pelo apoio financeiro concedido durante e para a concepção deste estudo.

\section{Referências}

AHMADI, F.; TAHROUDI, M.N.; MIRABBASI, R.; KHALILI, K.; JHAJHARIA, D. Spatiotemporal trend and abrupt change analysis of temperature in Iran. Meteorological Applications, v. 25, n. 2, p. 314-321, 2018.

ALEXANDER, L.V. Global observed long-term changes in temperature and precipitation extremes: A review of progress and limitations in IPCC assessments and beyond. Weather and Climate Extremes, v. 11, p. 4-16, 2016.

ALLEN, R.G.; PEREIRA, L.S.; RAES, D.; SMITH, M. Crop Evapotranspiration: Guidelines for Computing Crop Water Requirements-FAO irrigation and Drainage. Paper 56 Fao, Rome, 300, D05109, 1998.

ALVALA, R.C.S.; CUNHA, A.P.M.A.; BRITO, S.S.B.; SELUCHI, M.E.; MARENGO, J.A.; MORAES, O.L.L.; CARVALHO, M.A. Drought monitoring in the Brazilian Semiarid region. Anais da Academia Brasileira de Ciências, v. 91, n. 1, p. 1-15, 2017.

ANDREA, M.C.S.; DALLACORT, R.; BARBIERI, J.D.; TIEPPO, R.C. Impacts of Future Climate Predictions on Second Season Maize in an Agrosystem on a Biome Transition Region in Mato Grosso State. Revista Brasileira de Meteorologia, v. 34, n. 2, p. 335 - 347, 2019.

CABRAL JÚNIOR, J.B.; SANTOS E SILVA, C.M.; ALMEIDA, H.A.; BEZERRA, B.G.; SPYRIDES, M.H.C. Detecting linear trend of reference evapotranspiration in irrigated farming areas in Brazil's semiarid region. Theoretical and Applied Climatology, v. 138, n. 1-2, p. 215-225, 2019.

CARVALHO, M.A.V.; OYAMA, M.D. Atlantic Intertropical Convergence Zone width and intensity variability: observational aspects. Revista Brasileira de Meteorologia, v. 28, n. 3 , p. $305-316,2013$. 
CARVALHO, M.J.; MELO-GONÇALVES, P.; TEIXEIRA, J.C.; ROCHA, A. Regionalization of Europe based on a KMeans Cluster Analysis of the climate change of temperatures and precipitation. Physics and Chemistry of the Earth, Parts A/B/C, v. 94, p. 22-28, 2016.

CAVALCANTI, I.F.A.; FERREIRA, N.J.; SILVA, M.G.A.J.; DIAS, M.A.F.S. (2009). Tempo e Clima no Brasil. Oficina de Textos: São Paulo, Brazil, 2009.

CHATTOPADHYAY, N.; HULME, M. Evaporation and potential evapotranspiration in India under conditions of recent and future climate change. Agricultural and Forest Meteorology, v. 87, n. 1, p. 55-73, 1997.

COSTA, R.L.; BAPTISTA, G.M.M.; GOMES, H.B.; SILVA, F.D.S.; DA ROCHA JÚNIOR, R.L.; SALVADOR, M.A.; HERDIES, D.L. Analysis of climate extremes indices over northeast Brazil from 1961 to 2014. Weather and Climate Extremes, v. 28, p. 100254, 2020.

CUNHA, A.P.M.A.; ALVALÁ, R.C.S.; NOBRE, C.A.; CARVALHO, M.A. Monitoring vegetative drought dynamics in the Brazilian semiarid Region. Agricultural and Forest Meteorology, v. 214-215, p. 494-505, 2015.

CUNHA, A.P.M.A.; TOMASELLA, J.; RIBEIRO-NETO, G.G.; BROWN, M.; GARCIA, S.R.; BRITO, S.B.; CARVALHO, M.A. Changes in the spatial-temporal patterns of droughts in the Brazilian Northeast. Atmospheric Science Letters, v. 19, e855, 2018.

CUNHA, A.P.M.A.; ZERI, M.; LEAL, K.D.; COSTA, L.; CUARTAS, L.A.; MARENGO, J.A.; TOMASELLA, J.; VIEIRA, R.M.; BARBOSA, A.A.; CUNNINGHAM, C.; GARCIA, J.V.C.; BROEDEL, E.; ALVALÁ, R.; RIBEIRO-NETO, G. Extreme Drought Events over Brazil from 2011 to 2019. Atmosphere, v. 10, n. 642, 2019.

DA ROCHA JÚNIOR, R.L.; DOS SANTOS SILVA, F.D.; LISBOA COSTA, R.; BARROS GOMES, H.; HERDIES, D.L.; RODRIGUES DA SILVA, V.P.; CANDIDO XAVIER, A. Analysis of the Space-Temporal Trends of Wet Conditions in the Different Rainy Seasons of Brazilian Northeast by Quantile Regression and Bootstrap Test. Geosciences, v. 9, n. 11, p. 457, 2019.

DA ROCHA JÚNIOR, R.L.; DOS SANTOS SILVA, F.D.; LISBOA COSTA, R.; BARROS GOMES, H.; PINTO, D.D.C.; HERDIES, D.L. Bivariate Assessment of Drought Return Periods and Frequency in Brazilian Northeast Using Joint Distribution by Copula Method. Geosciences, v. 10, n. 4, p. 135, 2020.

DEL RÍO, S.; FRAILE, R.; HERRERO, L.; PENAS, A. Analysis of recent trends in mean maximun and minimun temperatures in a region of the NW of Spain (Castilla y León). Theoretical and Applied Climatology, v. 90, n. 1, p. 1-12, 2007.

DINPASHOH, Y.; BABAMIRI, O. Trends in reference crop evapotranspiration in Urmia Lake basin. Arabian Journal of Geosciences, v. 13, n. 372, p. 1-16, 2020.

DOS REIS, L.C.; SANTOS E SILVA, C.M.; BEZERRA, B.G.; MUTTI, P.R.; SPYRIDES, M.H.C.; DA SILVA, P.E. Analysis of Climate Extreme Indices in the MATOPIBA Region, Brazil. Pure and Applied Geophysics, v. 177, n. 4, p. 4457-4478, 2020a.
DOS REIS, L.C.; SANTOS E SILVA, C.M.; BEZERRA, B.G.; SPYRIDES, M.H.C. Caracterização da variabilidade da precipitação no MATOPIBA, região produtora de soja. Revista Brasileira de Geografia Física, v. 13, n. 4, p. 1425-1441, 2020b.

DOUVILLE, H.; RIBES, A.; DECHARME, B.; ALKAMA, R.; SHEFFIELD, J. Anthropogenic influence on multidecadal changes in reconstructed global evapotranspiration. Nature Climate Change, v. 3, n. 1, p. 59-62, 2012.

DUFEK, A.S.; AMBRIZZI, T. Precipitation variability in São Paulo State, Brazil. Theoretical and Applied Climatology, v. 93, n. 3, p. 167-178, 2008.

FISCHER, E.M.; KNUTTI, R. Anthropogenic contribution to global occurrence of heavy-precipitation and high-temperature extremes. Nature Climate Change, v. 5, n. 6, p. 1-6, 2015.

GHAFOURI-AZAR, M.; BAE, D-H.; KANG, S-U. Trend Analysis of Long-Term Reference Evapotranspiration and Its Components over the Korean Peninsula. Water, v. 10, n. 10, p. 1373, 2018.

GRIESER, J.; TROMEL, S.; SCHÖNWIESE, C-D. Statistical time series decomposition into significant components and application to European temperature. Theoretical and Applied Climatology, v. 71, n. 3, p. 171-183, 2002.

HAYLOCK, M.R.; PETERSON, T.C.; ALVES, L.M.; AMBRIZZI, T.; ANUNCIAÇÃO, Y.M.T.; BAEZ, J.; BARROS, V.R.; BERLATO, M.A.; BIDEGAIN, M.; CORONEL, G.; CORRADI, V.; GARCIA, V.J.; GRIMM, A.M.; KAROLY, D.; MARENGO, J.A.; MARINO, M.B.; MONCUNILL, D.F.; NECHET, D.; QUINTANA, J.; REBELLO, E.; RUSTICUCCI, M.; SANTOS, J.L.; TREBEJO, I.; VINCENT, L.A. Trends in Total and Extreme South American Rainfall in 1960-2000 and Links with Sea Surface Temperature. Journal of Climate, v. 19, n. 8, p. $1490-1512,2006$.

HUNTINGTON, T.G. Evidence for intensification of the global water cycle: review and synthesis. Journal of Hydrology, v. 319, n. 1-4, p. 83-95, 2006.

JENKINS, K.; WARREN, R. Quantifying the impact of climate change on drought regimes using the Standardised Precipitation Index. Theoretical and Applied Climatology, v. 120, n. 1-2, p. 41-54, 2015.

JIN, Z.; LIANG, W.; YANG, Y.; ZHANG, W.; YAN, J.; CHEN, $\mathrm{X}$.; LI, S.; MO, X. Separating vegetation greening and climate change controls on evapotranspiration trend over the Loess Plateau. Scientific Reports, v. 7, n. 1, p. 1-15, 2017.

JUNG, M.; REICHSTEIN, M.; CIAIS, P.; SENEVIRATNE, S.I.; SHEFFIELD, J.; GOULDEN, M.L.; BONAN, G.; CESCATTI, A.; CHEN, J.; DE JEU, R. Recent decline in the global land evapotranspiration trend due to limited moisture supply. Nature, v. 467, n. 7318, p. 951-954, 2010.

KENDALL, M.G. Rank Correlation Methods, 4th ed, Charles Griffin: London, 1975.

LIU, W.T.; JUÁREZ, R.I.N. ENSO drought onset prediction in northeast Brazil using NDVI. International Journal of Remote Sensing, v. 22, n. 17, p. 3483-3501, 2001.

LIU, X.; WANG, S.; ZHOU, Y.; WANG, F.; LI, W.; LIU, W. Regionalization and Spatiotemporal Variation of Drought in China Based on Standardized Precipitation Evapo- 
transpiration Index (1961-2013). Advances in Meteorology, v. 2015, n. 2, 2015.

LOPEZ-URREA, R.; MARTIN DE SANTA OLALLA, F.; FABEIRO, C.; MORATALLA, A. Testing evapotranspiration equations using lysimeter observationsin a semiarid climate. Agricultural Water Management, v. 85, n. 1-2, p. 15-26, 2006.

MACHIWAL, D.; JHA, M.K. Comparative Evaluation of Statistical Tests for Time Series Analysis: Application to Hydrological Time Series. Hydrological Sciences Journal, v. 53, n. 2, p. 353-366, 2008.

MACIEL, A.M.; VINHAS, L.; CAMARA, G. Algoritmos de clustering para separação de culturas agrícolas e tipos de uso e cobertura da Terra utilizando dados de sensoriamento remoto. Anais XVII Simpósio Brasileiro de Sensoriamento Remoto (SBSR), João Pessoa-PB, Brasil, 25 a 29 de abril de 2015, INPE.

MANN, H.B. Non-parametric tests against trend. Econometrica, v, 13, n. 9, p. 245-259, 1945.

MARENGO, J.A.; TORRES, R.R.; ALVES, L.M. Drought in Northeast Brazil-past, present, and future. Theoretical and Applied Climatology, v. 129, n. 3-4, p. 1189-120, 2016.

MARENGO, J.A.; ALVES, L.M.; ALVALÁ, R.C.S.; CUNHA, A.P.M.A.; BRITO, S.S.B.; MORAES, O.L.L. Climatic characteristics of the 2010-2016 drought in the semiarid Northeast Brazil region. Anais da Academia Brasileira de Ciências, v. 90, n. 2, p. 1973-1985, 2018.

MARTINS, E.S.P.R.; COELHO, C.A.S.; HAARSMA, R.; OTTO, F.E.L.; KING, A.D.; VAN OLDENBORGH, G.J.; KEW, S.; PHILIP, S.; JÚNIOR, F.C.V.; CULLEN, H. A multimethod attribution analysis of the prolonged northeast Brazil hydrometeorological drought (2012-16). Bulletin of the American Meteorological Society, v. 99, n. 1, p. 6569, $2018 \mathrm{a}$.

MARTINS, M.A.; TOMASELLA, J.; RODRIGUEZ, D.A.; ALVALÁ, R.C.; GIAROLLA, A.; GAROFOLO, L.L.; JÚNIOR, J.L.S.; PAOLICCHI, L.T.; PINTO, G.L. Improving drought management in the Brazilian semiarid through crop forecasting. Agricultural Systems, v. 160, p. 21-30, 2018b.

MEDEIROS, S.E.L.; ABRAHÃO, R.; GARCÍA-GARIZÁBAL, I.; IDMON MELO, B.M.P.; PEREIRA, L.S. Assessment of precipitation trends in the Sertão Paraibano Mesoregion. Revista Brasileira de Meteorologia, v. 33, n. 2, p. 344$352,2018$.

MODARRES, R.; SILVA, V.P.R. Rainfall trends in arid and semi-arid regions of Iran. Journal of Arid Environments, v. 70, n. 2, p. 344-355, 2001.

MORAES, J.M.; PELlEGRINO, G.Q.; BALLESTER, M.V.; MARTINELLI, L.A.; VICTORIA, R.L.; KRUSCHE, A.V. Trends in hydrological parameters of a southern Brazilian watershed and its relation to human induced changes. Water Resources Management, v. 12, n. 4, p. 295-311, 1998.

NECHET, D.; QUINTANA, J.; REBELLO, E.; RUSTICUCCI, M.; SANTOS, J.L.; TREBEJO, I.; VINCENT, L.A. Trends in total and extreme South American rainfall 1960-2000 and links with sea surface temperature. Journal of Climate, v. 19, n. 8, p. 1490-1512, 2006.
OKI, T.; KANAE, S. Global Hydrological Cycles and World Water Resources. Science, v. 313, n. 5790, p. 1068-1072, 2006.

OLIVEIRA, P.T.S.; NEARING, M.A.; MORAN, M.S.; GOODRICH, D.C.; WENDLAND, E.; GUPTA, H.V. Trends in water balance components across the Brazilian Cerrado. Water Resources Research, v. 50, n. 9, p. 7100-7114, 2014.

ONOZ, B.; BAYAZIT, M. The Power of Statistical Tests for Trend Detection. Turkish Journal of Engineering and Environmental Sciences, v. 27, n. 4, p. 247-251, 2003.

PAREDES-TREJO, F.; BARBOSA, H.; DOS SANTOS, C.A.C. Evaluation of the Performance of SM2RAIN-Derived Rainfall Products over Brazil. Remote Sensing, v. 11, n. 9, p. 1113, 2019.

PARTAL, T.; KAHYA, E. Trend analysis in Turkish precipitation data. Hydrological Processes, v. 20, n. 9, p. 2011-2026, 2006.

PAULINO, V.E.N.; STUDART, T.M.C.; CAMPOS, J.N.B.; PESTANA, C.J.; LUNA, R.M.; ALVES, J.M.B. Trends in Crop Reference Evapotranspiration and Climatological Variables Across Ceará State - Brazil. Revista Brasileira de Meteorologia, v. 34, n. 1, p. 79 - 88, 2019

RAES, D. The ETo Calculator. Food and Agriculture Organization of the United Nations (FAO): Rome, Italy, 2012.

RAZIEI, T.A. Precipitation regionalization and regime for Iran based on multivariate analysis. Theoretical and applied climatology, v. 131, p. 1429-1448, 2018.

REGONDA, S.K.; ZAITCHIK, B.F.; BADR, H.S.; RODELL, M. Using climate regionalization to understand Climate Forecast System Version 2 (CFSv2) precipitation performance for the Conterminous United States (CONUS). Geophysical Research Letters, v. 43, n. 12, p. 6485-6492, 2016.

RICHARD, O.G. Statistical methods for environmental pollution monitoring. John Wiley \& Sons, 1987.

RODRIGUES, R.R.; HAARSMA, R.J.; CAMPOS, E.J.D.; AMBRIZZI, T. The Impacts of Inter-El Niño Variability on the Tropical Atlantic and Northeast Brazil Climate. Journal of Climate, v. 24, n. 13, p. 3402-3422, 2011.

ROUSSEEUW, P.J. Silhouettes: a graphical aid to the interpretation and validation of cluster analysis. Journal of Computational and Applied Mathematics, v. 20, p. 53-65, 1987.

SALMI, T.; MÄÄTTÄ, A.; ANTTILA, P.; RUOHO-AIROLA, T.; AMNELL, T. Detecting trends of annual values of atmospheric pollutants by the Mann-Kendall test and Sen's slope estimates - the Excel template application MAKESENS. Helsink (Publications on Air Quality No. 31, Report code FMI-AQ-31), 2012.

SALVIANO, M.F.; GROPPO, J.D.; PELLEGRINO, G.Q. Análise de tendências em dados de precipitação e temperatura no Brasil. Revista Brasileira de Meteorologia, v. 31, n. 1, p. 64-73, 2016.

SANCHES, L.; VOURLITIS, G.L.; ALVES, M.C.; PINTOJÚNIOR, O.B.; NOGUEIRA, J.S. Seasonal patterns of evapotranspiration for a Vochysia divergens forest in the Brazilian Pantanal. Wetlands, v. 31, n. 6, p. 1215-1225, 2011. 
SANTOS, A.P.P.; ARAGÃO, M.R.S.; CORREIA, M.F.; SANTOS, S.R.Q.; SILVA, F.D.S.; ARAÚJO, H.Á. Precipitação na Cidade de Salvador: Variabilidade Temporal e Classificação em Quantis. Revista Brasileira de Meteorologia, v. 31, n. 4, p. 454-467, 2016.

SANTOS, C.A.C.; MARIANO, D.A.; NASCIMENTO, F.C.A.; DANTAS, F.R.C.; OLIVEIRA, G.; SILVA, M.T.; SILVA, L.L.; SILVA, B.B.; BEZERRA, B.G.; SAFA, B.; MEDEIROS, S.S.; NEALE, C.M.U. Spatio-temporal patterns of energy exchange and evapotranspiration during an intense drought for drylands in Brazil. International Journal of Applied Earth Observation and Geoinformation, v. 85, p. 101982, 2020.

SATYAMURTY, P.; CASTRO, A.A.; TOTA, J.; GULARTE, L.E.S.; MANZI, A.O. Rainfall trends in the Brazilian Amazon Basin in the past eight decades. Theoretical and Applied Climatology, v. 99, n. 1-2, 2010.

SEIDEL, E.J.; MOREIRA JÚNIOR, F.J.; ANSUJ, A.P.; NOAL, M.R.C. Comparação entre o método Ward e o método Kmédias noagrupamento de produtores de leite. Ciência e Natura, v. 30, n. 1, p. 7-15, 2008.

SEN, P.K. Estimates of the regression coefficient based on Kendall's tau. Journal of the American Statistical Association, v. 63, p. 1379-1389, 1968.

SILVA, V.B.S.; KOUSKY, V.E.; SILVA, F.D.S.; SALVADOR, M.A.; ARAVEQUIA, J.A. The 2012 severe drought over Northeast Brazil. Bulletin of the American Meteorological Society, v. 94, p. 162, 2013.

SILVA, F.D.S.; COSTA, R.L.; ANTONIO, M.A.V.; AFONSO, E.O.; SANTOS, D.M.; MATEUS, N.P.A.; ANTONIO, J.F. Tendências observadas da evapotranspiração potencial no Estado de Alagoas (1961-2016). Revista Brasileira de Geografia Física, v. 11, n. 1, p. 28-43, 2018.

SILVA, P.E.; SILVA, C.M.S.; SPYRIDES, M.H.C.; ANDRADE, L.M.B. Análise de Índices de Extremos Climáticos no Nordeste e Amazônia Brasileira para o Período entre 1980 a 2013. Anuário do Instituto de Geociências, v. 42, n. 2, p. 137-148, 2019.

SOME'E, B.S.; EZANI, A.; TABARI, H. Spatiotemporal trends of aridity index in arid and semi-arid regions of Iran. Theoretical and Applied Climatology, v. 111, n. 1-2, p. 149$160,2013$.

STAEGER, T.; GRIESER, J.; SCHÖNWIESE, C-D. Statistical separation of observed global and European climate data into natural and anthropogenic signals. Climate Research, v. 24, n. 1, p. 3-13, 2003.

TABARI, H.; MAROFI, S.; AEINI, A.; TALAEE, P.H.; MOHAMMADI, K. Trend analysis of reference evapo- transpiration in the western half of Iran. Agricultural and Forest Meteorology, v. 151, n. 2, p. 128-136, 2011.

THOMAS, A. Spatial and temporal characteristics of potential evapotranspiration trends over China. International Journal of Climatology, v. 20, n. 4, p. 381-396, 2000.

TOMASELLA, J.; VIEIRA, R.M.S.P.; BARBOSA, A.A.; RODRIGUEZ, D.A. Desertification trends in the Northeast of Brazil over the period 2000-2016. International Journal of Applied Earth Observation and Geoinformation, v. 73, 2018. DOI: 10.1016/j.jag.2018.06.012.

VENTURA, F.; SPANO, D.; DUCE, P.; SNYDER, R.L. An evaluation of common evapotranspiration equations. Irrigation Science, v. 18, n. 4, p. 163-170, 1999.

VOURLITIS, G.L.; FILHO, N.P.; HAYASHI, M.M.S.; NOGUEIRA, J.S.; CASEIRO, F.T.; CAMPELO, J.H. Seasonal variations in the evapotranspiration of a transitional tropical forest of Mato Grosso, Brazil. Water Resources Research, v. 38, n. 6, p. 1-11, 2002.

XAVIER, A.C.; KING, C.W.; SCANLON, B.R. Daily gridded meteorological variables in Brazil (1980-2013). International Journal of Climatology, v. 36, n. 6, p. 2644-2659, 2016.

WILKS, D.S. Statistical Methods in the Atmospheric Sciences. 3rd ed. Elsevier, 676 pg, 2011.

ZENG, Z.Z.; WANG, T.; ZHOU, F.; CIAIS, P.; MAO, J.F.; SHI, X.Y.; PIAO, S.L. A worldwide analysis of spatiotemporal changes in water balance-based evapotranspiration from 1982 to 2009. Journal of Geophysical Research: Atmospheres, v. 119, n. 3, p. 1186-1202, 2014.

ZHANG, D.J.; ZHANG, X.X.; PENG-FEI, W.U. Relationship between ET and LUCC in a typical watershed of Loess Plateau over the past 20 years. Arid Land Geography, v. 34, p. 400-408, 2011.

ZHANG, Y.; PEÑA-ARANCIBIA, J.L.; MCVICAR, T.R.; CHIEW, F.H.S.; VAZE, J.; LIU, C.; LU, X.; ZHENG, H.; WANG, Y.; LIU, Y.Y.; MIRALLES, D.G.; PAN, M. Multidecadal trends in global terrestrial evapotranspiration and its components. Scientific Reports, v. 6, n. 1, p. 1-12, 2016.

ZHANG, Y.; MOGES, S.; BLOCK, P. Optimal cluster analysis for objective regionalization of seasonal precipitation in regions of high spatial-temporal variability: application to Western Ethiopia. Journal of Climate, v. 29, n. 10, p. 3697-3717, 2016.

License information: This is an open-access article distributed under the terms of the Creative Commons Attribution License (type CC-BY), which permits unrestricted use, distribution and reproduction in any medium, provided the original article is properly cited. 